\title{
Nerve Fibres Detection in Paired Eutopic and Ectopic Endometria from Women with Endometriosis: Correlation with Nerve Growth Factor Expression
}

\author{
Sebastián Venegas 1,2, Edward Parada ${ }^{1}$, M. Angélica Boric ${ }^{1}$, Hugo Sovino ${ }^{1,3}$, \\ Ariel Fuentes 1,3, Fernando Gabler 3,4, Reinaldo González-Ramos ${ }^{1,3}$, M. Cecilia Johnson1 \\ ${ }^{1}$ Institute of Maternal and Child Research, Faculty of Medicine, University of Chile, Santiago, Chile \\ ${ }^{2}$ Medical Technology School, Faculty of Medicine, University of Chile, Santiago, Chile \\ ${ }^{3}$ San Borja Arriarán Clinical Hospital, Santiago, Chile \\ ${ }^{4}$ Department of Pathology, Faculty of Medicine, University of Chile, Santiago, Chile \\ Email: cjohnson@med.uchile.cl
}

Received 20 April 2015; accepted 26 July 2015; published 29 July 2015

Copyright (C) 2015 by authors and Scientific Research Publishing Inc.

This work is licensed under the Creative Commons Attribution International License (CC BY). http://creativecommons.org/licenses/by/4.0/

(c) (i) Open Access

\section{Abstract}

Endometriosis, disorder characterized by the presence of endometrium outside the uterus cavity, is associated with chronic pelvic pain. Nerve growth factor (NGF) participates in development, repair and survival of neurons. We evaluated NGF and nerve fibres in eutopic and ectopic endometria from women with endometriosis and in normal endometrium from control patients without endometriosis. In endometrium collected during surgery for endometriosis (paired eutopic and ectopic endometria, proliferative $n=6$ and secretory $n=7$ phases) or during tubal ligation or hysterectomy for no endometrial disease (control, proliferative $n=6$ and secretory $n=6$ phases), NGF and neurofilament (NF) of nerve fibres were studied by immunohistochemistry measuring integrated optical density (IOD). Cytoplasmic NGF was detected in glands and stroma in all control, eutopic and ectopic endometrial samples; no statistical differences were found throughout the menstrual cycle. However, total (gland plus stroma) NGF IOD was higher in eutopic than in ectopic endometria during the secretory phase. NF was detected only in ectopic endometrium being eutopic and control endometria negative to this antibody, and no differences were observed between proliferative and secretory phases. Negative and significant correlation was found between NGF and NF expression in ectopic endometria only during the secretory phase. In conclusion, our results show a significant negative correlation between NGF and myelinated nerve fibres in secretory ectopic endometria. NGF expression was not significantly modified throughout the menstrual cycle in control and in endometriosis, even higher in eutopic than in ectopic tissues; the NGF secretion from lesions to pelvic cavity cannot be ruled out, and could explain the negative correla- 
tion observed. Although NF and NGF are not useful as diagnostic markers for endometriosis, their expression may be related to endometrial physiology and pathophysiology of the disease.

\title{
Keywords
}

\author{
NGF, Nerves Fibres, Eutopic Endometrium, Ectopic Endometrium, Endometriosis
}

\section{Introduction}

Endometriosis is a gynaecologic disorder characterized by the presence of endometrial tissue outside the uterus cavity denominated as ectopic endometrium. Until now, the aetiology of this old disease is unclear, being the retrograde menstruation/transplantation theory the most accepted [1]-[3]. The most common localizations of the endometriotic lesions are in the peritoneum, the ovaries, the uterosacral ligaments and rectovaginal septum [4].

In patients with endometriosis, dysmenorrhea, dyspareunia, dyschezia and chronic pelvic pain are frequent and produced by unknown mechanisms. The inflammatory condition is a consequence of the synthesis and release of several proinflammatory mediators from endometriotic lesions and immune cells to peritoneal fluid in the pelvic cavity and participates on pain and infertility associated to endometriosis [5] [6]. The high macrophage concentration reported in the peritoneal fluid from these patients is related with the increased nerve fibres density in the endometriotic lesions which are activated by the pro-inflammatory molecules secreted by the same macrophages [7] [8].

The presence of nerve fibres in endometriosis patients has been shown in eutopic (intrauterine) and ectopic endometria, including ovarian endometriomas, peritoneal endometriotic lesions and deep infiltrating endometriosis, but almost undetectable in control endometria from healthy women [9]-[11]. This important finding leads to the proposal to use nerve fibres detection as a diagnostic method through minimal invasive biopsy of eutopic endometria [12]-[14].

An elevated concentration of nerve growth factor (NGF) has been detected in peritoneal fluid from endometriosis women [15]. This peptide is structural and functionally related to the neurotrophins, a family with an important role in the development, differentiation and neuronal survival in both central and peripheral nervous systems. NGF is synthesized by various cell types including immune system, smooth muscle, cells of endocrine system as epithelial and non-neuronal ovarian cells [16]-[18]. In rat uterus, the NGF is related with innervations during the estrous cycle and estrogen positively regulates its synthesis [19] [20]. In human, information of NGF in healthy endometrium and eutopic endometrium from endometriosis women is scarce being the most reports in ectopic endometrium including peritoneal endometriotic lesions and deep infiltrating (DIE) endometriosis [21]-[25].

Our objectives were to detect nerve fibres and NGF in healthy endometrium and in paired eutopic and ectopic endometria from Chilean women with endometriosis; and also to study the correlation between nerve fibres and NGF expressions.

\section{Materials and Methods}

\subsection{Subjects}

Endometrium (functional layer) obtained with a Pipelle suction curette from the uterine corpus, and ectopic endometrium removed during the surgery at the Clinical Hospital San Borja Arriarán from women undergoing laparoscopy for tubal sterilization or hysterectomy for no endometrial disease (controls, $n=12,25-46$ years) or for endometriosis (endometriosis, $n=13,22$ - 46 years). All patients signed informed consent; the institutional committee boards approved this study. Both eutopic and ectopic endometria were obtained from the same patient. The women did not receive hormonal treatment for at least 2 months before surgery.

Eutopic and ectopic endometrial samples were transported in ice-cold phosphate buffered saline (PBS) to laboratory where were washed with cold PBS. A portion was included in formalin/PBS for histological evaluation and immunohistochemistry. An experimental pathologist (FG) classified the endometria according to Noyes criteria in proliferative (days 6 - 14, 6 control and 6 endometriosis) and secretory (days 15 - 24, 6 control and 7 
endometriosis) phases of the menstrual cycle and confirmed the presence of endometria in the endometriotic lesions in all samples.

\subsection{Immunohistochemistry}

Immunohistochemistry staining was performed on $4 \mu \mathrm{m}$ formalin-fixed sections as described in Johnson et al. (2005) [26] with some modifications. Briefly, after deparaffinized with xylol and rehydrated through decreasing grade alcohol, all slides were treated with $1 \mathrm{mmol} / \mathrm{L}$ EDTA pH 8 in steamer for 20 min, quenched with $6 \% \mathrm{H}_{2} \mathrm{O}_{2}$ in methanol and blocked with $2 \%$ bovine serum albumin (BSA) in PBS for $1 \mathrm{~h}$ at room temperature. Then, the slides were incubated in humidify chamber with antibodies against to NGF (1:200, rabbit polyclonal antibody (ab6199) Abcam, Cambridge, UK) and to neurofilament (NF), specific marker for myelinated nerve fibres (1: 800, mouse monoclonal antibody (2F11), Cell Marque, Rocklin, CA, USA) at $37^{\circ} \mathrm{C}$ for $1 \mathrm{~h}$. The immunodetection was performed using streptavidin-biotin peroxidase system (ABC RTU Vectastain kit, Vector Laboratories, Burlingame, CA, USA) for 30 min, then, diaminobenzidine as a chromogen (Vector Laboratories) and hematoxylin as a counterstaining. Human spinal medulla was used as positive control and incubation with $2 \%$ BSA in PBS without the first antibody as negative control. The staining was evaluated by digitalization using the integrated optical density (IOD) tool from the Image Pro 6.3 software (Olympus, Melville, NY, USA) performed in at least 10 microphotographs from each sample obtained with Optical microscope Olympus BX51 and digital camera Q imaging Micro Publisher 3.3 RTV (Olympus). The illumination condition and amplification were similar in all measurements.

\subsection{Statistical Analysis}

Results are expressed in boxplot, which depicts numeral data through their quartiles, and whiskers vertical lines extended from the box indicating the data variability outside the upper and lower quartiles. For continuous variables was used Mann Whitney or Kruskal-Wallis followed by Dunn test. Spearman test was used for correlation study. The significance level was set at 5\%. The age and BMI are expressed as the means \pm SEM. GraphPad Prism 4.0 (GraphPath Software, San Diego, CA, USA) was used to conduct statistical analysis.

\section{Results}

\subsection{Antropometric and Endometriosis Characteristic}

No significant differences were found in age $(p=0.098)$ and body mass index (BMI; $p=0.524)$ between control and endometriosis women. Nine of the endometriosis patients had chronic pelvic pain and one had dysmenorrhea; in three patients with endometriosis and in all control women these symptoms were not assessed. The endometriosis stage (American Society for Reproductive Medicine, 1997) was III (43\%) and IV (57\%) and the lesions were localized in one or both ovaries in eleven patients, in the rectovaginal septum as nodules (RVN) in two patients, and concomitantly with these lesions in uterosacral ligaments (four with ovarian endometriosis and one with RVN) and in peritoneum (five with ovarian endometriosis and one with RVN) (Table 1).

\subsection{Endometrial NGF Immunohistochemistry}

NGF was strongly expressed throughout the menstrual cycle as a heterogeneous staining with small granulations in the gland and stromal cytoplasm of endometrium from control women (Figure 1(A) and Figure 1(B)), and from endometriosis women: eutopic endometrium (Figure 1(D) and Figure 1(E)) and ectopic endometrium in ovary (Figure 1(G) and Figure 1(H)), in peritoneum (Figure 1(J)), in RVN (Figure 1(K)) and in inflammatory cells morphologically indentified as macrophages localized in ovarian, uterosacral ligaments and peritoneal lesions (Figure 1(M), Figure $1(\mathrm{~N})$ and Figure $1(\mathrm{O})$ ).

A constant NGF IOD was measured in the gland (Figure 2(A)) and stroma (Figure 2(B)) from control endometria during the proliferative and secretory phases. High dispersion data were obtained in the gland throughout the menstrual cycle and in the secretory stroma in eutopic and ectopic endometria from endometriosis women. No significant differences were observed between control and eutopic or ectopic endometria or between eutopic and ectopic endometria during the menstrual cycle. When the total positive NGF staining was analyzed, the IOD in eutopic endometrium was significantly higher than ectopic endometrium during the secretory phase (Figure 2(C)). 
Table 1. Subjects anthropometric and endometriosis characteristics.

\begin{tabular}{|c|c|c|c|c|c|}
\hline & & \multicolumn{4}{|c|}{ Patients } \\
\hline \multirow{2}{*}{\multicolumn{2}{|c|}{ Menstrual Cycle }} & \multicolumn{2}{|c|}{ Endometriosis $(\mathrm{n}=13)$} & \multicolumn{2}{|c|}{ Control $(n=12)$} \\
\hline & & $\begin{array}{l}\text { Proliferative Phase } \\
\qquad(\mathrm{n}=6)\end{array}$ & $\begin{array}{l}\text { Secretory Phase } \\
\quad(\mathrm{n}=7)\end{array}$ & $\begin{array}{l}\text { Proliferative Phase } \\
\qquad(\mathrm{n}=6)\end{array}$ & $\begin{array}{l}\text { Secretory Phase } \\
\quad(n=6)\end{array}$ \\
\hline \multicolumn{2}{|c|}{ Age (Years) } & \multicolumn{2}{|c|}{$32.6 \pm 2.2$} & \multicolumn{2}{|c|}{$37.6 \pm 1.8 p=0.098$} \\
\hline \multicolumn{2}{|c|}{ BMI $\left(\mathrm{Kg} / \mathrm{m}^{2}\right)$} & \multicolumn{2}{|c|}{$23.7 \pm 1.0$} & \multicolumn{2}{|c|}{$24.6 \pm 1.0 p=0.524$} \\
\hline \multicolumn{2}{|c|}{ Pain $(n=10 ; N I=15)$} & $\mathrm{n}=4 ; \mathrm{NI}=2$ & $\mathrm{n}=6 ; \mathrm{NI}=1$ & $\mathrm{NI}=6$ & $\mathrm{NI}=6$ \\
\hline \multirow{5}{*}{$\begin{array}{c}\text { Endometriosis Stages } \\
\text { (ASRM, 1997) }\end{array}$} & Stage III & 3 & 3 & & \\
\hline & Stage IV & 3 & 4 & & \\
\hline & Ovary & 4 & 7 & & \\
\hline & RVN & 1 & 1 & & \\
\hline & Ovary + USL & 1 & 3 & & \\
\hline \multirow[t]{4}{*}{ Lesion Localization } & Ovary + RVN & 1 & 0 & & \\
\hline & Ovary + Peritoneum & 2 & 3 & & \\
\hline & RVN + USL & 1 & 0 & & \\
\hline & RVN + Peritoneum & 1 & 0 & & \\
\hline
\end{tabular}

Patients without (control, $n=12$ ) or with $(n=13)$ endometriosis were classified in proliferative or secretory phases of the menstrual cycle. Ten patients with endometriosis have pain, but this symptom was not assessed in 3 endometriosis patients and in all control patients. RVN: Rectovaginal nodules. USL: Uterosacral ligaments. BMI: body mass index. NI: no information.

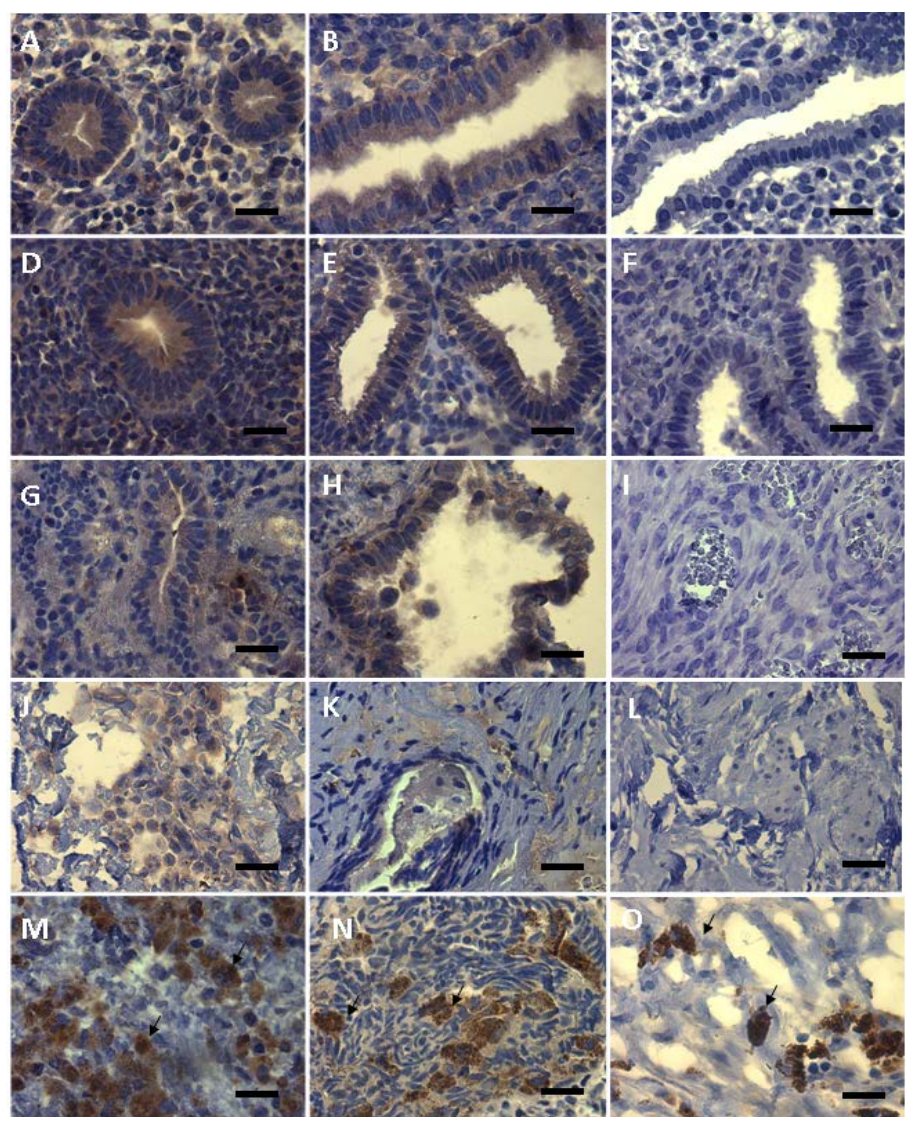

Figure 1. Immunohistochemical staining of NGF in eutopic and ectopic endometria during the proliferative and secretory phases. Representative healthy endometrium from control women (A)-(C) and eutopic (D)-(F) and ectopic endometria from women with endometriosis (G)-(O). Epithelial and/or stromal cells in endometriotic lesions in ovary (G) (H), peritoneum (J), rectovaginal nodule $(\mathrm{RVN}$; $(\mathrm{K})$ ). Black arrows indicate positive NGF staining in morphological like macrophage cells in ovary (M), uterosacral ligaments (n) and peritoneum (O). Proliferative phase (A), (D), (G), (J), (M), (O) and secretory phase (B), (E), (H), (K), (N). Negative control (C), (F), (I), (L). Diaminobenzidine was used as chromogen and hematoxylin as a counterstaining. Barr $50 \mu \mathrm{m}$. 

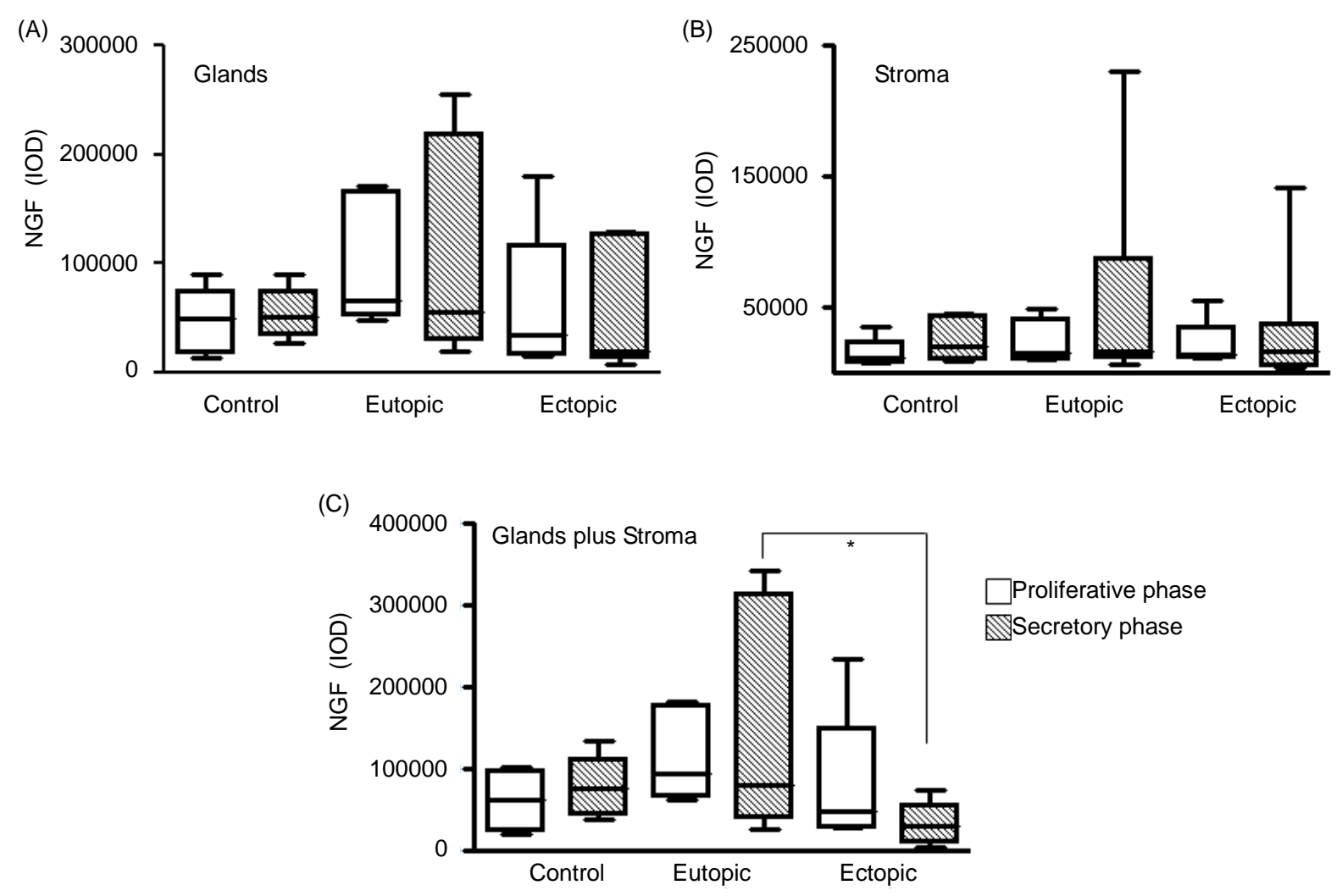

Figure 2. Boxplot of IOD immunostaining of NGF protein in endometrial epithelial (A) and stromal (B) cells, and total NGF IOD (C) from control and endometriosis women during the menstrual cycle. Healthy (control), and eutopic and ectopic (endometriosis) endometria were obtained during the surgery. Proliferative phase: control $(n=6)$, eutopic $(n=6)$ and ectopic $(n$ $=6)$ endometria. Secretory phase: control $(n=6)$; eutopic $(n=7)$ and ectopic $(n=7)$ endometria. IOD: Integrated optical density.

\subsection{Nerve Fibres Immunohistochemistry}

Positive brown staining for nerve fibres using NF antibody was detected in ectopic endometria from 12 endometriosis women (Figures 3(E)-(K)); ten of these patients reported pain; we did not have pain information from the other two cases. Only in one patient with ovarian endometriosis the NF staining was negative and the pain antecedent was not available. Nerve fibres were not detected in control endometria or in eutopic endometria from endometriosis patients throughout the menstrual cycle (Figures 3(A)-(D)).

No significant differences were found in nerve fibres IOD analyzed in ectopic endometria during the menstrual cycle (Figure 4).

\subsection{NGF and NF Correlation}

Significant negative correlation between NF and NGF in ectopic endometria was found during the secretory phase (Spearman $r=-0.8857, p=0.033$ ), but not during the proliferative phase (Spearman $r=-0.20, p=0.71$ ).

\section{Discussion}

In this study, we showed the presence of NGF in paired eutopic and ectopic endometria from women with endometriosis as well as in healthy endometria throughout the menstrual cycle. Nerve fibres were detected only in ectopic endometria from endometriosis women, and negative correlation between NGF and nerve fibres was found during the secretory phase.

Our results confirmed the presence of NGF in glandular and stromal endometrial cell compartments both from control and endometriosis women as previously was reported [25] [27] [28]. Recently, we communicated higher NGF mRNA expression in eutopic endometrium in women with RVN endometriosis than with ovarian 


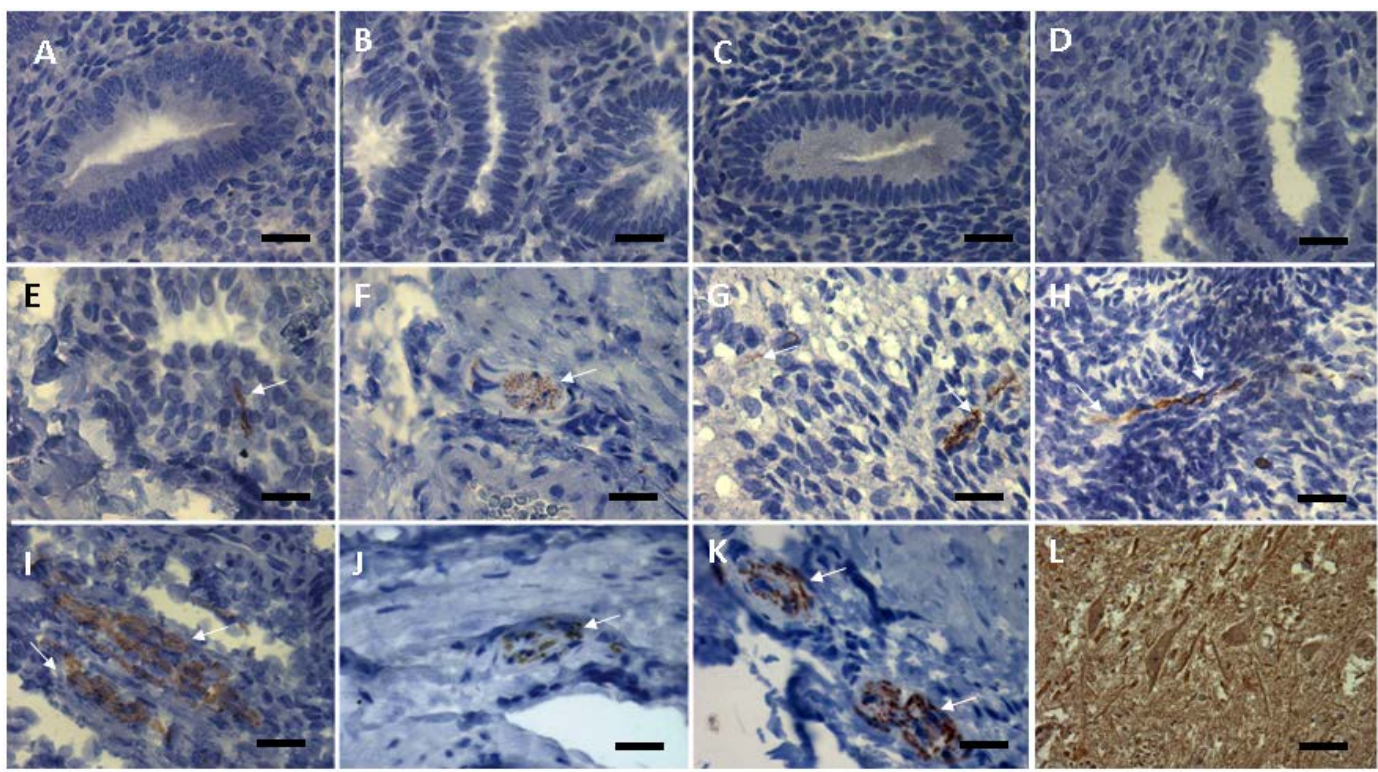

Figure 3. Immunohistochemical staining of nerve fibre neurofilaments in ectopic endometria during the proliferative and secretory phases. Representative healthy endometrium from control women (A) and (B), and eutopic (C) and (D) and ectopic (E)-(K) endometria from women with endometriosis. Positive NF in endometriotic lesions in peritoneum $(\mathrm{E})$ and $(\mathrm{F})$, ovary $(\mathrm{G})-(\mathrm{I})$, rectovaginal nodule $(\mathrm{RVN}$; $(\mathrm{J})$ ) and uterosacral ligaments (K). Positive control: spinal cord (L). White arrows indicate positive NF nerve fibres. Diaminobenzidine was used as chromogen and hematoxylin as a counterstaining. Barr $50 \mu \mathrm{m}$.

Ectopic Endometria

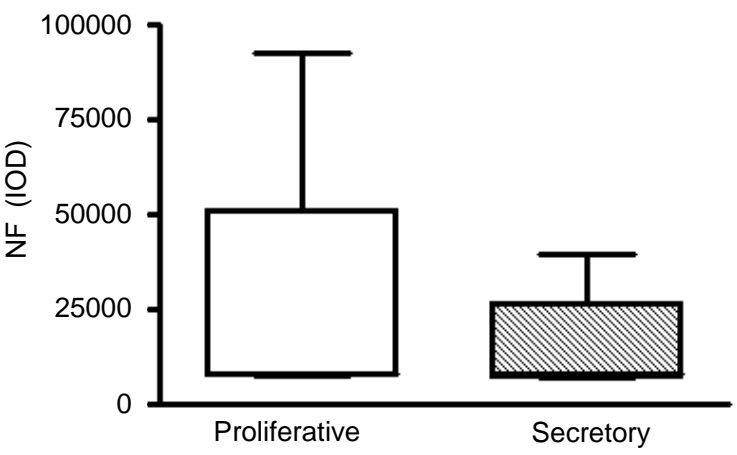

Figure 4. Boxplot of IOD immunostaining of nerve fibres with neurofilament (NF) in endometrial explants from ectopic endometria during the menstrual cycle. NF detection was performed in ectopic endometria obtained from ovarian $(n=11)$ and RVN $(n=2)$ lesions. IOD: Integrated optical density. Proliferative phase $n$ $=6$. Secretory phase $n=6 .^{*} p<0.05$ Mann Whitney test.

endometriosis [29]. In the present study, we observed elevated NGF expression in eutopic and ectopic endometrial glands, although not statistically different to healthy endometria, probably as a consequence of the heterogeneity of the endometriosis group, which included ovarian and RVN endometriosis some of them combined with peritoneal or uterosacral ligament lesions. In the present, peritoneal, ovarian and rectovaginal endometriosis are being considered as different entities as several authors propose [30]-[32], so to increase the sample number and to separate them according to localization of the lesion is a pending challenge. Besides, grouping the samples according early, middle and late proliferative and secretory phases would allow a more detailed analysis during the menstrual cycle. Recently, Mita et al. [33] reported in endometrial cell lines the inhibitory effect of progesterone through its receptors on NGF expression, aspect that we cannot observe during the secretory phase in this study.

Similar levels of NGF protein and mRNA expressions were reported in eutopic endometria from women with 
and without endometriosis [25] [27], but lower levels in control endometria compared to endometriotic lesions [28]. As our knowledge, this is the first study with paired eutopic and ectopic endometria during the menstrual cycle. The finding of the reduced NGF expression in ectopic compared to its paired eutopic endometria was an unexpected result. At this respect, Kajitani et al. [28] proposed that endometriotic lesions not only synthesize NGF, but also secrete it to the pelvic cavity, coincidently with the high NGF concentration in the peritoneal fluid from these patients [15].

In this study, several cells with granular aspect and morphologically identified as macrophages, strongly expressed NGF in ovarian, peritoneal, uterosacral ligaments and RVN ectopic endometria, which is in agreement with the reported macrophages presence very close to endometriotic cells described in peritoneal endometriosis [8]. Consequently, NGF may induce the release of inflammatory cytokines from immune cells in the pelvic cavity which in turn may induce the synthesis of this growth factor [33] [34]. In addition, the known estrogenic microenvironment in eutopic and ectopic endometria in women with endometriosis [3] [34]-[36] may be also involved in the induction of the NGF expression in macrophages as Kanda et al. [37] reported. However, to confirm which types of immune cells are present in these tissues are necessary further studies.

Controversial results are reported respect to the presence of nerve fibres in eutopic endometria from endometriosis women. Nerve fibres are also detected in endometria from other pathologies such as adenomyosis, uterine fibroids and adhesions, particularly associated with pain [38]-[40]. It is known the strong association of endometriosis with pelvic pain, also confirmed in this study, in which all the patients that referred severe pain had NF positive nerve fibres in ectopic endometria. Our results using the NF antibody, which recognized myelinated nerve fibres [41], show their presence in ectopic endometria, but not in the functional layer of eutopic endometria from women with and without endometriosis. Similarly to us, Tokushige et al. [21] using NF antibody did not detect these myelinated nerve fibres in these tissues, at difference to Bokor et al. [12] who detected them in a few eutopic endometrial samples from endometriosis (5\%) and control (10\%) women. The neural protein usually detected in eutopic endometrium is PGP9.5 by a pan antibody, which recognizes both myelinated and non myelinated nerve fibres [8] [12]-[14] [42]. Both results suggest the lack of myelinated nerve fibres in the functional layer of eutopic endometrium.

The eutopic endometrium expresses several abnormal genes and once in the pelvic cavity presents the capacity of implantation, invasion, growth and neoangiogenesis similarly to neoplasia [43]-[45]. In this pelvic inflammatory microenvironment, the development of the endometriotic lesions is favoured, as also of the nerve fibres. The peritoneal fluid from endometriosis women induces the development of thick bundles of nerves fibres in chicken dorsal root ganglia, effect not reproduced by peritoneal fluid from control women [46].

Furthermore, NGF, besides to stimulate the nerve fibres development, may be involved in the pain associated with endometriosis. This factor may induce the nerve stimulus conduction in pain sensitive conducer fibres [47]. On the other hand, NGF and its precursor proNGF co-exist in vivo, but NGF is rapidly secreted by the cells, unlike proNGF, which is retained in the cell cytoplasm [48]-[50]. The significant negative correlation found between NGF and NF in ectopic endometria during the secretory phase supports the idea that in this type of endometria NGF predominate over pro-NGF. We cannot rule out the possibility of higher proNGF presence in eutopic than ectopic endometria, considering the dual specificity of the NGF antibody that recognized both, mature and immature molecules. It will be necessary to analyze others nerve fibres markers with others antibodies.

The strength of this work is the study of both paired eutopic and ectopic endometria from the same patient and their comparison with control endometria; unfortunately, the detection of only two antigens and the reduced number of samples are weaknesses.

In synthesis, our results show a significant negative correlation between NGF and myelinated nerve fibres in secretory ectopic endometria. NGF expression was not significantly modified throughout the menstrual cycle in control and in endometriosis, even higher in eutopic than in ectopic tissues; the NGF secretion from lesions to pelvic cavity cannot be ruled out, and could explain the negative correlation observed. Although NF and NGF are not useful as diagnostic markers for endometriosis, their expression may be related to endometrial physiology and pathophysiology of the disease.

\section{Acknowledgements}

The authors are grateful to the women who donated tissue, as without their generous contributions, this study would not have been possible. This work was supported by Fondo Nacional de Ciencias y Tecnología 
(FONDECYT) \#1120074, Santiago, Chile.

\section{Disclosure Summary}

The authors have nothing to disclose.

\section{References}

[1] Sampson, J. (1927) Peritoneal Endometriosis Due to the Menstrual Dissemination of Endometrial Tissue into the Peritoneal Cavity. American Journal of Obstetrics \& Gynecology, 14, 422-469.

[2] Giudice, L.C. and Kao, L.C. (2004) Endometriosis. Lancet, 364, 1789-1799. http://dx.doi.org/10.1016/S0140-6736(04)17403-5

[3] Bulun, S.E. (2009) Endometriosis. New England Journal of Medicine, 360, 268-279. http://dx.doi.org/10.1056/NEJMra0804690

[4] Burney, R.O. and Giudice, L.C. (2012) Pathogenesis and Pathophysiology of Endometriosis. Fertility and Sterility, 98, 511-519. http://dx.doi.org/10.1016/j.fertnstert.2012.06.029

[5] Halme, J., Becker, S. and Haskill, S. (1987) Altered Maturation and Function of Peritoneal Macrophages: Possible Role in Pathogenesis of Endometriosis. American Journal of Obstetrics \& Gynecology, 156, 783-789. http://dx.doi.org/10.1016/0002-9378(87)90333-4

[6] McKinnon, B., Bersinger, N.A., Wotzjow, C. and Mueller, M.D. (2012) Endometriosis-Associated Nerve Fibers, Peritoneal Fluid Cytokine Concentrations, and Pain in Endometriotic Lesions from Different Locations. Fertility and Sterility, 97, 373-380. http://dx.doi.org/10.1016/j.fertnstert.2011.11.011

[7] Kyama, C.M., Debrock, S., Mwenda, J.M. and D’Hooghe, T.M. (2003) Potential Involvement of the Immune System in the Development of Endometriosis. Reproductive Biology and Endocrinology, 1, 123. http://dx.doi.org/10.1186/1477-7827-1-123

[8] Tran, L.V.P., Tokushige, N., Berbic, M., Markham, R. and Fraser, I.S. (2009) Macrophages and Nerve Fibers in Peritoneal Endometriosis. Human Reproduction, 24, 835-841. http://dx.doi.org/10.1093/humrep/den483

[9] Anaf, V., Simon, P., El Nakadi, I., Fayt, I., Simonart, T., Buxant, F. and Noël, J.C. (2002) Hyperalgia, Nerve Infiltration and Nerve Growth Factor Expression in Deep Adenomyotic Nodules, Peritoneal and Ovarian Endometriosis. Human Reproduction, 17, 1895-1900. http://dx.doi.org/10.1093/humrep/17.7.1895

[10] Tokushige, N., Markham, R., Russell, P. and Fraser, I.S. (2006) Nerve Fibers in Peritoneal Endometriosis. Human Reproduction, 21, 3001-3007. http://dx.doi.org/10.1093/humrep/del260

[11] Kobayashi, H., Yamada, Y., Morioka, S., Niiro, E., Shigemitsu, A. and Ito, F. (2014) Mechanism of Pain Generation for Endometriosis-Associated Pelvic Pain. Archives of Gynecology and Obstetrics, 289, 13-21. http://dx.doi.org/10.1007/s00404-013-3049-8

[12] Bokor, A., Kyama, C.M., Vercruysse, L., Fassbender, A., Gevaert, O., Vodolazkaia, A., De Moor, B., Fülöp, V. and D’Hooghe, T. (2009) Density of Small Diameter Sensory Nerve Fibres in Endometrium: A Semi-Invasive Diagnostic test for Minimal to Mild Endometriosis. Human Reproduction, 24, 3025-3032. http://dx.doi.org/10.1093/humrep/dep283

[13] Al-Jefout, M., Dezarnaulds, G., Cooper, M., Tokushige, N., Luscombe, G.M., Markham, R. and Fraser, I.S. (2009) Diagnosis of Endometriosis by Detection of Nerve Fibers in an Endometrial Biopsy: A Double Blind Study. Human Reproduction, 24, 3019-3024. http://dx.doi.org/10.1093/humrep/dep275

[14] Aghaey Meibody, F., Mehdizadeh Kashi, A., Zare Mirzaie, A., Ghajarie Bani Amam, M., Shariati Behbahani, A., Zolali, B. and Najafi, L. (2011) Diagnosis of Endometrial Nerve Fibers in Women with Endometriosis. Archives of Gynecology and Obstetrics, 284, 1157-1162. http://dx.doi.org/10.1007/s00404-010-1806-5

[15] Barcena de Arellano, M.L., Arnold, J., Vercellino, F., Chiantera, V., Schneider, A. and Mechsner, S. (2011) Overexpression of Nerve Growth Factor in Peritoneal Fluid from Women with Endometriosis May Promote Neurite Outgrowth in Endometriotic Lesions. Fertility and Sterility, 95, 1123-1126. http://dx.doi.org/10.1016/j.fertnstert.2010.10.023

[16] Levi-Montalceni, R. (1987) The Nerve Growth Factor 35 Years Later. Science, 237, 1154-1162. http://dx.doi.org/10.1126/science.3306916

[17] Ansel, J.C., Kaynard, A.H., Armstrong, C.A., Olerud, J., Bunett, N. and Payan, D. (1996) Skin Nervous System Interactions. Journal of Investigative Dermatology, 106, 198-204. http://dx.doi.org/10.1111/1523-1747.ep12330326

[18] Dissen, G.A., Parrott, J.A., Skinner, M.K., Hill, D.F., Costa, M.E. and Ojeda, S.R. (2000) Direct Effects of Nerve Growth Factor on Thecal Cells from Antral Ovarian Follicles. Endocrinology, 141, 4736-4750. 
http://dx.doi.org/10.1210/en.141.12.4736

[19] Chalar, C., Richeri, A., Viettro, L., Chavez-Genaro, R., Bianchimano, P., Marmol, N.M. and Crutcher, K. (2003) Plasticity in Developing Rat Uterine Sensory Nerves: The Role of NGF and TrkA. Cell and Tissue Research, 314, 191-205. http://dx.doi.org/10.1007/s00441-003-0799-9

[20] Shi, Z.Q., Arai, K.Y., Jin, W.Z., Weng, Q., Watanabe, G., Suzuki, A.K. and Taya, K.S. (2006) Expression of Nerve Growth Factor and Its Receptors NTRK1 and TNFRSF1B Is Regulated by Estrogen and Progesterone in the Uteri of Golden Hamsters. Biology of Reproduction, 74, 850-856. http://dx.doi.org/10.1095/biolreprod.105.044917

[21] Tokushige, N., Markham, R., Russsell, P. and Fraser, I.S. (2006) High Density of Small Nerve Fibres in the Functional Layer of the Endometrium in Women with Endometriosis. Human Reproduction, 21, 782-787. http://dx.doi.org/10.1093/humrep/dei368

[22] Tokushige, N., Markham, R., Russell, P. and Fraser, I.S. (2008) Effects of Hormonal Treatment on Nerve Fibers in Endometrium and Myometrium in Women with Endometriosis. Fertility and Sterility, 90, 1589-1598. http://dx.doi.org/10.1016/j.fertnstert.2007.08.074

[23] Wang, G., Tokushige, N., Markham, R. and Fraser, I. (2009) Rich Innervation of Deep Infiltrating Endometriosis. Human Reproduction, 24, 827-834. http://dx.doi.org/10.1093/humrep/den464

[24] Arnold, J., Barcena de Arellano, M.L., Ruster, C., Vercellino, G.F., Chiamtara, V., Schneider, A. and Mechsner, S. (2012) Imbalance between Sympathetic and Sensory Innervation in Peritoneal Endometriosis. Brain, Behavior, and Immunity, 26, 132-141. http://dx.doi.org/10.1016/j.bbi.2011.08.004

[25] Browne, A.S., Yu, J., Huang, R.-P., Francisco, A.M.C., Sidell, N. and Taylor, R.N. (2012) Proteomic identification of Neurotrophins in the Eutopic Endometrium of Women with Endometriosis. Fertility and Sterility, 98, 713-719. http://dx.doi.org/10.1016/j.fertnstert.2012.05.027

[26] Johnson, M.C., Torres, M., Alves, A., Bacallao, K., Fuentes, A., Vega, M. and Boric, M.A. (2005) Augmented Cell Survival in Eutopic Endometrium from Women with Endometriosis: Expression of c-myc, TGF-Beta 1 and Bax Genes. Reproductive Biology and Endocrinology, 3, 45. http://dx.doi.org/10.1186/1477-7827-3-45

[27] Barcena de Arellano, M.L., Arnold, J., Sacher, F., Blochler, M., Staube, M., Bartley, J., Vercellino, F., Chiantera, V., Schneidr, A. and Mechsner, S. (2012) Eutopic Endometrium from Women with Endometriosis Does Not Exhibit Neurotrophic Properties. Journal of Neuroimmunology, 249, 49-55. http://dx.doi.org/10.1016/j.jneuroim.2012.04.020

[28] Kajitani, T., Maruyama, T., Asada, H., Uchida, H., Oda, H., Uchida, S., Miyazaki, K., Arase, T., Ono, M. and Yoshimura, Y. (2013) Possible Involvement of Nerve Growth Factor in Dysmenorrhea and Dyspareunia Associated with Endometriosis. Journal of Endocrinology, 60, 1155-1164. http://dx.doi.org/10.1507/endocrj.ej13-0027

[29] Johnson, M.C., Parada, E., Molina, C., Castro, J., Gabler, F., Sovino, H., Martínez, A., González-Ramos, R., Boric, M.A. and Fuentes, A. (2015) Positive Correlation between NGF, TRKA or p75NTR Levels and Disease Stages in Eutopic Endometrium from Endometriosis Women. 16th Human Reproduction World Congress, Berlin, 18-21 March 2015.

[30] Donnez, J., Nisolle, M., Snoes, P., Gillet, N., Beguin, S. and Casanas-Roux, F. (1996) Peritoneal Endmetriosis and Endometriotic Nodules of the Rectovaginal Septum Are Two Different Entities. Fertility and Sterility, 66, 362-368.

[31] Matsuzaki, S., Maleysson, E. and Darcha, C. (2010) Analysis of Matrix Metalloproteinase-7 Expression in Eutopic and Ectopic Endometrium Samples from Patients with Different Forms of Endometriosis. Human Reproduction, 25, 742750. http://dx.doi.org/10.1093/humrep/dep435

[32] Aghajanova, L. and Giudice, L.C. (2011) Molecular Evidence for Differences in Endometrium in Severe versus Mild Endometriosis. Reproductive Sciences, 18, 229-251. http://dx.doi.org/10.1177/1933719110386241

[33] Mita, S., Shimizu, Y., Sato, A., Notsu, T., Imada, K. and Kyo, S. (2014) Dienogest Inhibits Nerve Growth Factor Expression Induced by Tumor Necrosis Factor- $\alpha$ or Interleukin-1 $\beta$. Fertility and Sterility, 101, 575-601. http://dx.doi.org/10.1016/j.fertnstert.2013.10.038

[34] Noble, L.S., Simpson, E., Johns, A. and Bulun, S.E. (1996) Aromatase Expression in Endometriosis. The Journal of Clinical Endocrinology \& Metabolism, 81, 174-179.

[35] Johnson, M.C., Pinto, C., Alves, A., Palomino, A., Fuentes, A., Boric, M.A. and Vega, M. (2004) P450Arom and Estrogenic Microenvirontment of Eutopic Endometria in Endometriosis. Revista médica de Chile, 132, 1475-1482.

[36] Bulun, E.S., Monsavais, D., Pavone, M.E., Dyson, M., Xue, Q., Attar, E., Tokunaga, H. and Su, E.J. (2014) Role of Estrogen Receptor- $\beta$ in Endometriosis. Nature Medicine, 18, 1016-1018.

[37] Kanda, N. and Watanabe, S. (2003) 17Beta-Estradiol Enhances the Production of Nerve Growth Factor in THP1-Derived Macrophages or Peripheral Blood Monocyte-Derived Macrophages. Journal of Investigative Dermatology, 121, 771-780. http://dx.doi.org/10.1046/j.1523-1747.2003.12487.x

[38] Zhang, X., Lu, B., Huang, X., Xu, H., Zhou, C. and Lin, J. (2009) Endometrial Nerve Fibers in Women with Endometriosis, Adenomyosis, and Uterine Fibrois. Fertility and Sterility, 92, 1799-1801. 
http://dx.doi.org/10.1016/j.fertnstert.2009.05.016

[39] Zhang, X., Lu, B., Huang, X., Xu, H., Zhou, C. and Lin, J. (2010) Innervation of Endometrium and Myometrium in Women with Painful Adenomyosis and Uterine Fibroids. Fertility and Sterility, 94, 730-737. http://dx.doi.org/10.1016/j.fertnstert.2009.03.026

[40] Barcena de Arellano, M.L., Wagner, M.F., Oldeweme, J., Arnold, J., Ebert, A., Schneider, A. and Mechsnere, S. (2012) Neurotrophin Expression Is Not Affected in Uteri of Women with Adenomiosis. Journal of Molecular Neuroscience, 47, 495-504. http://dx.doi.org/10.1007/s12031-012-9757-x

[41] Schlaepfer, W.W. (1987) Neurofilaments: Structure, Metabolism and Implications in Disease. Journal of Neuropathology \& Experimental Neurology, 46, 117-129. http://dx.doi.org/10.1097/00005072-198703000-00001

[42] Zevallos, H.B.-V., McKinnon, B., Tokushige, N., Mueller, M.D., Fraser, I.S. and Bersinger, N.A. (2015) Detection of the Pan Neuronal Marker PGP9.5 by Immunohistochemistry and Quatitative PCR in Eutopic Endometrium from Women with and without Endometriosis. Archives of Gynecology and Obstetrics, 291, 85-91. http://dx.doi.org/10.1007/s00404-014-3379-1

[43] Taylor, R.N., Ryan, I.P., Moore, E.S., Hornung, D., Shifren, J.L. and Tseng, J.F. (1997) Angiogenesis and Macrophage Activation in Endometriosis. Annals of the New York Academy of Sciences, 828, 194-207. http://dx.doi.org/10.1111/j.1749-6632.1997.tb48540.x

[44] Bruner, K.L., Eisenberg, E., Gorstein, F. and Osteen, K.G. (1999) Progesterone and Transforming Growth Factor-Beta Coordinately Regulate Suppression of Endometrial Matrix Metalloproteinases in a Model of Experimental Endometriosis. Steroids, 64, 648-653. http://dx.doi.org/10.1016/S0039-128X(99)00048-3

[45] Cho, S., Young, S.C., Youn, E.J., Kyung, J., Choi, Y.M, Yim, S.Y., Kim, H.Y., Seo, S.K. and Lee, B.S. (2012) Expression of Vascular Endothelial Growth Factor (VEGF) and Its Soluble Receptor-1 in Endometriosis. Microvascular Research, 83, 237-242. http://dx.doi.org/10.1016/j.mvr.2011.12.004

[46] Barcena de Arellano, M.L., Arnold, J., Lang, H., Vercellino, G.F., Chiantera, V., Schneider, A. and Mechsner, S. (2013) Evidence of Neurotrophic Events Due to Peritoneal Endometriotic Lesions. Cytokine, 62, 253-261. http://dx.doi.org/10.1016/j.cyto.2013.03.003

[47] Zhang, G., Dmitrieva, N., Liu, Y., McGinty, K.A. and Berkley, K.J. (2008) Endometriosis as a Neurovascular Condition: Estrous Variations in Innervations, Vascularisation, and Growth Factor Content of Ectopic Endometrial Cyst in the Rat. The American Journal of Physiology-Regulatory, Integrative and Comparative Physiology, 294, R162-R171. http://dx.doi.org/10.1152/ajpregu.00649.2007

[48] Lee, R., Kermani, P., Teng, K.K. and Hempstead, B.L. (2001) Regulation of Cell Survival by Secreted proneurotrophins. Science, 294, 1945-1948. http://dx.doi.org/10.1126/science.1065057

[49] Arici, I., D’Onofrio, M., Brandi, R., Malerba, F., Paoletti, F., Storti, A.E., Florenzano, F., Fasulo, L. and Cattaneo, A. (2014) ProNGF/NGF Mixture Induce Gene Expression Changes in PC12 Cells that Neither Singly Produces. BMC Neuroscience, 15, 48. http://dx.doi.org/10.1186/1471-2202-15-48

[50] Choi, S. and Friedman, W.J. (2014) Interleukin-1 $\beta$ Enhances Neuronal Vulnerability to ProNGF-Mediated Apoptosis by Increasing Surface Expression of p75NTR and Sortillin. The Journal of Neuroscience, 257, 11-19. http://dx.doi.org/10.1016/j.neuroscience.2013.10.058 